\title{
AN OSCILLATORY DIFFERENTIAL EQUATION
}

\section{S. P. Hastings and J. B. McLeod}

ABSTRACT. The paper discusses the equation

$$
y^{\prime \prime}+e^{-x+y^{2}} y=0
$$

and gives a complete discussion of the asymptotic behavior of solutions, showing in particular that there are both oscillating and non-oscillating solutions.

\section{Introduction}

In the context of a review of oscillatory and non-oscillatory equations, J. S. W. Wong has raised the following question:

Is the equation

$$
y^{\prime \prime}+e^{-x+y^{2}} y=0, \quad 0<x<\infty
$$

oscillatory or non-oscillatory?

The background to this question lies in a conjecture made by F. V. Atkinson in the early 80 's and which appeared in print for the first time in [1]. This concerned the equation

$$
y^{\prime \prime}+y \exp \left(|y|^{m}-x\right)=0, \quad m>0,
$$

and the conjecture was that this contains only non-oscillatory solutions if $0<m<2$, but both oscillatory and non-oscillatory if $m \geq 2$. In [1] the conjecture was proved for $0<m<2$, and the current paper proves the conjecture for the critical case $m=2$. We understand from J. S. W. Wong that he now has extended the ideas of the present paper to deal with $m>2$ and thus completed the proof of the Atkinson conjecture.

Here we show that the equation (1.1) possesses both oscillatory and non-oscillatory solutions, and we are able to go further by obtaining the complete asymptotic behavior, as $x \rightarrow \infty$, of all solutions of the equation. In the remainder of this section, we describe heuristically what the situation is and state the precise theorems. In later sections we provide the rigorous proofs.

If we multiply (1.1) by $y^{\prime}$ and integrate, we have

$$
\frac{1}{2} y^{\prime 2}+\frac{1}{2} e^{-x+y^{2}}+\frac{1}{2} \int^{x} e^{-t+y^{2}} d t=\text { const. }
$$

so that $y^{\prime 2}+e^{-x+y^{2}}$ is positive decreasing, and so is bounded. Thus, for any solution,

$$
\begin{gathered}
y^{\prime} \text { is bounded, } \\
y^{2}-x \text { is bounded above, } \\
\int^{\infty} e^{-t+y^{2}} d t<\infty
\end{gathered}
$$

Received February 16, 1996.

1991 Mathematics Subject Classification: 34C05, 34C10, 34C15, 34D05.

Key words and phrases: ordinary differential equation, limit-cycle, oscillatory, asymptotics. 
From (1.5) it is clear that $y^{2}-x$ should be large and negative for most $x$ as $x \rightarrow \infty$, and (1.1) then suggests that $y^{\prime \prime}$ is small so $y^{\prime}$ is essentially constant. Thus as $x \rightarrow \infty$, we expect that the solution consists of straight line segments, each segment continuing until it comes close to the curve $y^{2}=x$, after which the solution "bounces back" onto another straight line segment. Whether the solution is oscillatory or not depends on whether these successive segments cross the line $y=0$ or not.

Computer studies of (1.1) show some solutions which oscillate initially, but the space between successive zeros of $y$ increases rapidly. Hence it is unclear from these studies if the oscillations continue. However, these computer investigations, plus the obvious importance of the curve $y^{2}=x$, led us to make the change of variables

$$
u=x^{-1 / 2} y, \quad v=x^{1 / 2} y^{\prime} \text {. }
$$

Then the equations for $u$ and $v$ are

$$
\begin{aligned}
& x u^{\prime}=v-\frac{1}{2} u \\
& x v^{\prime}=\frac{1}{2} v-x^{2} u e^{x\left(u^{2}-1\right)},
\end{aligned}
$$

and when this system is studied numerically and solutions plotted in the $(u, v)$-plane, we see strong evidence for the existence of both oscillating and non-oscillating solutions.

These last equations certainly suggest that most of the time, as $x \rightarrow \infty$, we have

$$
x u^{\prime}=v-\frac{1}{2} u, \quad x v^{\prime}=\frac{1}{2} v,
$$

which has the first integral

$$
(u-v) v=\text { const. }
$$

This suggests that we should look at the quantity $(u-v) v$ in the context of the full equations (1.6), (1.7), and in fact these can be written in the form

$$
\frac{d}{d x}\left\{(u-v) v-x e^{y^{2}-x}\right\}=-\left(y^{2}-x+1\right) e^{y^{2}-x} .
$$

Now from (1.5) and the fact that $y^{2}-x$ is bounded above, we can conclude that

$$
\int^{x}\left(y^{2}-x+1\right) e^{y^{2}-x} d x
$$

either converges as $x \rightarrow \infty$ or diverges to $-\infty$. Thus

$$
(u-v) v-x e^{y^{2}-x} \rightarrow L,
$$

say, as $x \rightarrow \infty$, where possibly $L=+\infty$. However, we know already from (1.4) that $u$ is bounded, and so the left-hand side of (1.11), if it is unbounded, can only be unbounded negative. Thus, in fact, $L$ is finite, and we can conclude that

$$
\begin{aligned}
v=x^{1 / 2} y^{\prime} & \text { is bounded, } \\
x e^{y^{2}-x} & \text { is bounded. }
\end{aligned}
$$

Since it is presumably the case that $x e^{y^{2}-x}$ is small most of the time as $x \rightarrow \infty$, we will have $(u-v) v$ close to $L$. (At the moment, this is only a heuristic observation, to be made more precise and proved below.) We therefore look now at the phase-plane curves given by

$$
(u-v) v=L
$$




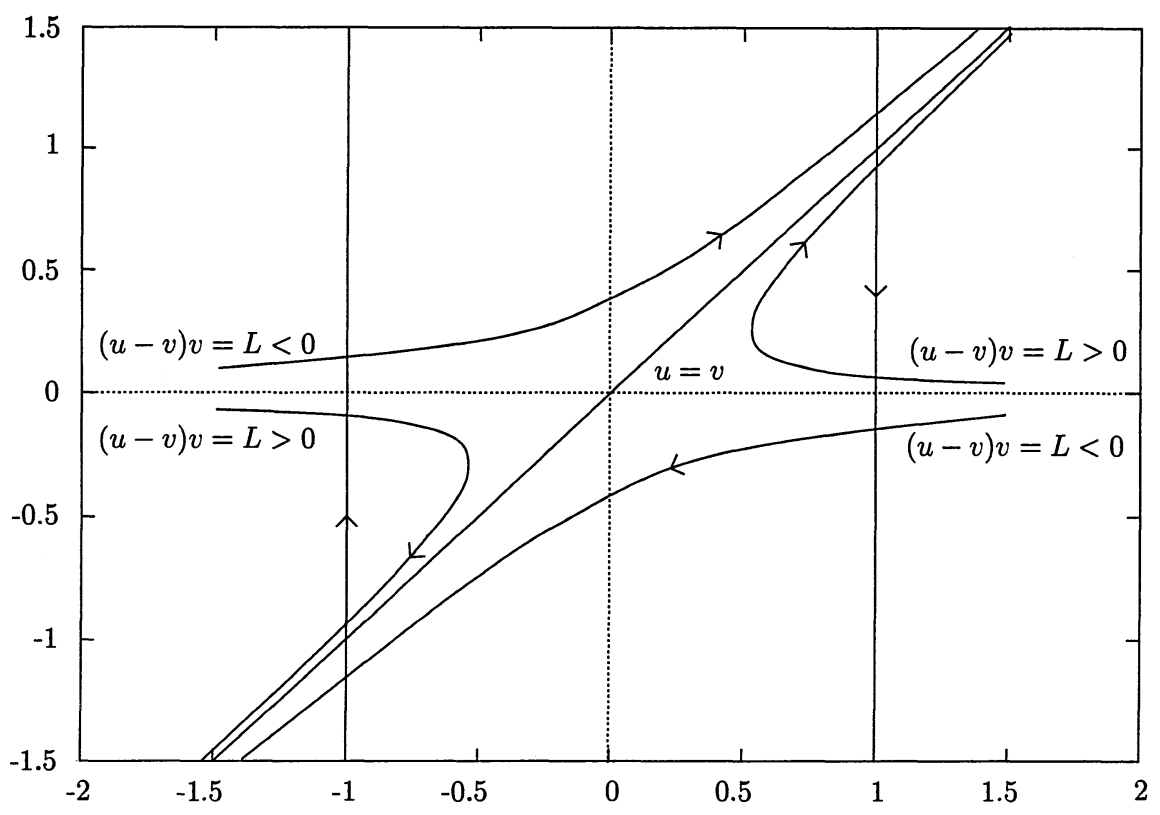

FIGURE 1

For each $L$, the curve is a hyperbola, with two disjoint branches. For $L=0$, the hyperbola degenerates to straight lines. However, there is the added restriction in the full problem that from (1.13), we always have $y^{2} \leq x, u^{2} \leq 1$, so that, as $x \rightarrow \infty$, what happens is that the solution proceeds along a branch of a hyperbola until it reaches $u= \pm 1$, when it moves along the line $u= \pm 1$ to the other branch of the same hyperbola $(L<0)$ or to another point on the same branch $(L>0)$. It thus tends to a singular limit-cycle consisting of segments of a hyperbola intercepted by segments of the lines $u= \pm 1$. Note also that necessarily $L \leq \frac{1}{4}$, since $L>\frac{1}{4}$ implies that the curve represented by $(u-v) v=L$ lies entirely to the right of $u=1$ or entirely to the left of $u=-1$.

The limiting behavior of $y$ as a function of $x$ can, of course, be obtained from this. It consists, as already said, of segments of straight lines (when in the phase plane we are on a branch of a hyperbola) interrupted by "bounces" from the curve $y^{2}=x$ (when the solution is on the line $u= \pm 1$ and $v$ in effect has a discontinuity). If $L<0$, the solution is oscillatory, since $u$ changes sign on the hyperbola. If $L>0$, the solution is non-oscillatory, and the solution keeps bouncing back from the hyperbola, but never crosses the $x$-axis.

We are now in a position to state rigorous theorems.

Theorem 1. $\left(L \neq 0, \frac{1}{4}\right)$ (i) Let $C_{L}$ denote the limit curve assembled above, for $0<L<\frac{1}{4}$ or $L<0$. Then, if $d\left\{(u, v), C_{L}\right\}$ denotes the distance in the phase plane of the solution $(u, v)$ from $C_{L}$ where $L$ is related to the solution through (1.11), we have

$$
d\left\{(u, v), C_{L}\right\}=O(\log x / x)
$$


(ii) If $L<0$, there exists a sequence of values $\left\{x_{n}\right\}, x_{n} \sim k a_{L}^{n}$ for some positive constants $k, a_{L}(>1)$, such that, for $x_{n} \leq x \leq x_{n+1}$,

$$
y(x) \sim \frac{(-1)^{n}}{x_{n+1}-x_{n}}\left\{\sqrt{x_{n}}\left(x_{n+1}-x\right)-\sqrt{x_{n+1}}\left(x-x_{n}\right)\right\},
$$

and we have the relation

$$
a_{L}=(\sqrt{1-4 L}+1)^{4} /\left(16 L^{2}\right)
$$

(iii) If $0<L<\frac{1}{4}$, there exists a sequence of values $\left\{x_{n}\right\}, x_{n} \sim k a_{L}^{n}$ for some positive constants $k, a_{L}(>1)$, such that, for $x_{n} \leq x \leq x_{n+1}$,

$$
y(x) \sim \pm \frac{1}{x_{n+1}-x_{n}}\left\{\sqrt{x_{n}}\left(x_{n+1}-x\right)+\sqrt{x_{n+1}}\left(x-x_{n}\right)\right\}
$$

the sign, for any particular solution $y$, being either always positive or always negative. Further, we again have the relation (1.15).

Finally, there are solutions for every $L<\frac{1}{4}, L \neq 0,1 / 4$.

Theorem 2. $(L=0)$ The limit set $C_{0}$ consists of two triangles, one in the first quadrant, one in the third, with the origin as the only common point. As in Theorem $1, d\left\{(u, v), C_{0}\right\} \rightarrow 0$, and in the phase plane the solution either converges to $(0,0)$ or to the whole of $C_{0}$, i.e., traversing each half alternately.

If the solution converges to $(0,0)$, then $y \rightarrow A$, for some constant $A$.

Both types of solutions exist.

Theorem 3. $\left(L=\frac{1}{4}\right)$ The limit set $C_{1 / 4}$ consists of the two points $\left(1, \frac{1}{2}\right),\left(-1,-\frac{1}{2}\right)$. We have $y \sim x^{1 / 2}$ or $y \sim-x^{1 / 2}$, and there is precisely one solution with each behavior.

While this answers the original question about oscillatory behavior (and more), one could go on, using the ideas in the proof of the above theorems, to say more about the asymptotic behavior of the solutions near the "bounce" points $x_{n}$, or about the asymptotic positions of the points $x_{n}$, but we do not pursue this further here.

It is also the case that the methods described here would apply more generally to equations of the form

$$
y^{\prime \prime}+e^{f(y)-g(x)} y=0
$$

but again we do not pursue this.

\section{Proof of Theorem 1}

We prove first that $d\left\{(u, v), C_{L}\right\} \rightarrow 0$ as $x \rightarrow \infty$. From (1.5), we see that there must exist a sequence of points $x_{n}^{*} \rightarrow \infty$ such that

$$
\lim _{n \rightarrow \infty} x_{n}^{*} e^{y^{2}-x_{n}^{*}}=0 .
$$

(For otherwise, $e^{y^{2}-x} \geq K x^{-1}$ for some positive constant $K$ and all sufficiently large $x$, and this contradicts (1.5).) Hence there must be a corresponding sequence $\left(u_{n}, v_{n}\right)$ where $u_{n}=u\left(x_{n}^{*}\right), v_{n}=v\left(x_{n}^{*}\right)$, such that $\left(u_{n}-v_{n}\right) v_{n} \rightarrow L$, so that $\left(u_{n}, v_{n}\right)$ is close to $C_{L}$. If $\left|u_{n}\right| \nrightarrow 1$, then it is clear from (1.6), (1.7) that, for $x>x_{n}^{*}$, the point $(u, v)$ will remain close to $C_{L}$ until $u$ is close to \pm 1 (say, without loss of generality, $u=+1$ ). It is also clear that, as $x \rightarrow \infty, \lim \sup |u| \leq 1$, since the contrary would contradict (1.4) or (1.13). Thus, if $(u, v)$ is close to $C_{L}$ and $u$ close to 1 , then $v$ is close to a root of

$$
v^{2}-v+L=0
$$


i.e.,

$$
v=\frac{1}{2}(1 \pm \sqrt{1-4 L})
$$

and since we have reached this point with increasing $x$, the arrows on Figure 1 show that in fact

$$
v=\frac{1}{2}(1+\sqrt{1-4 L})>\frac{1}{2} .
$$

Thus from (1.6), $u$ continues to increase (and thus remains close to 1) until $v$ decreases to $\frac{1}{2}$. Hence $(u, v)$ remains close to $C_{L}$ at $u=1, v=\frac{1}{2}$, and it then follows that at this point

$$
x e^{y^{2}-x} \sim \frac{1}{4}-L>0
$$

So long as $x^{2} e^{y^{2}-x}$ remains large, it follows from (1.7) that $v$ continues to decrease, and, since now $v<\frac{1}{2} u$, also $u$ decreases. Thus $v$ is still decreasing when $x e^{y^{2}-x}$ is small, and then $(u-v) v$ is close to $L$, and $(u, v)$ is close to $C_{L}$, although now on the other branch of $C_{L}(L<0)$ or at the other end of the same branch (if $0<L<\frac{1}{4}$ ). Then, as $x$ further increases, $(u, v)$ moves again along the hyperbolic segment of $C_{L}$. This discussion shows that as $x \rightarrow \infty$, we must have

$$
d\left\{(u, v), C_{L}\right\} \rightarrow 0 .
$$

To prove the more precise estimate (1.14), we first note that if we define

$$
H=y^{2}-x+\log x,
$$

then, as $x \rightarrow \infty, H$ is bounded above (an immediate consequence of (1.13)) with a sequence of maxima at points $x_{n}$, say. It is clear that the maxima must occur when $(u, v)$ is close to one of the straight line segments of $C_{L}$ and that at them, $H$ is bounded below, from (2.1). Further, since

$$
H^{\prime}=2 y y^{\prime}-1+\frac{1}{x}=2 u v-1+\frac{1}{x}
$$

it is clear that, at $x_{n}, u \sim 1, v \sim \frac{1}{2}$ (or $u \sim-1, v \sim-\frac{1}{2}$ ), and by considering $H^{\prime \prime}$, it is easy to see that there is precisely one maximum near $\left(1, \frac{1}{2}\right)$ and one near $\left(-1,-\frac{1}{2}\right)$ in each circuit of $C_{L}$. These are the points $x_{n}$. Of course, if $L>0$, the maxima occur only near $\left(1, \frac{1}{2}\right)$, or alternatively only near $\left(-1,-\frac{1}{2}\right)$, since the solution does not oscillate.

We next compute the distance $x_{n+1}-x_{n}$. We may suppose without loss of generality that $y\left(x_{n}\right)>0$. Since

$$
H^{\prime \prime}=-2 \frac{y^{2}}{x} e^{H}+2 y^{\prime 2}-\frac{1}{x^{2}},
$$

we have

$$
H^{\prime \prime}+2 \frac{y^{2}}{x} e^{H}=O\left(\frac{1}{x}\right) .
$$

Thus, if

$$
e^{H} \geq \log x / x^{2} \quad \text { for } \quad x_{n} \leq x \leq x_{n}^{*}
$$


which defines $x_{n}^{*}$, we have, for $x_{n} \leq x \leq x_{n}^{*}$,

$$
\begin{aligned}
H^{\prime \prime} & \sim-2 e^{H}, \\
\frac{1}{4} H^{\prime 2} & \sim e^{H_{0}}-e^{H}, \quad H_{0}=H\left(x_{n}\right),
\end{aligned}
$$

so that, on integration,

$$
e^{-\frac{1}{2} H_{0}} \cosh ^{-1}\left(e^{\frac{1}{2} H_{0}-\frac{1}{2} H}\right) \sim x-x_{n} .
$$

Since

$$
\cosh ^{-1} y \sim \log y \quad \text { for large } y
$$

we have

$$
\begin{aligned}
x_{n}^{*}-x_{n} & \sim \frac{1}{2} e^{-\frac{1}{2} H_{0}}\left(H_{0}-H\right)\left(x_{n}^{*}\right) \\
& \sim \frac{1}{2} e^{-\frac{1}{2} H_{0}} \log \left(x_{n}^{* 2} / \log x_{n}^{*}\right) \\
& \sim e^{-\frac{1}{2} H_{0}} \log x_{n} \\
& \sim \frac{2 \log x_{n}}{\sqrt{1-4 L}} \text { from }(2.1) .
\end{aligned}
$$

Similarly, if $x_{n+1}^{*}$ is such that (2.2) holds for $x_{n+1}^{*} \leq x \leq x_{n+1}$, we have

$$
x_{n+1}-x_{n+1}^{*} \sim \frac{2 \log x_{n+1}}{\sqrt{1-4 L}}
$$

For $x_{n}^{*} \leq x \leq x_{n+1}^{*}$, we have

$$
e^{H}=O\left(\frac{\log x_{n}}{x_{n}^{2}}\right)
$$

so that

$$
(u-v) v-L=o(1) .
$$

Thus, from (1.7), with $t=\log x, t_{n}^{*}=\log x_{n}^{*}, t_{n+1}^{*}=\log x_{n+1}^{*}$, we have

$$
\frac{d v}{d t}=\frac{1}{2} v+O\left(\frac{\log x_{n}}{x_{n}}\right)
$$

and since, from $(2.5), u^{2}\left(x_{n}^{*}\right)=1+O\left(\log x_{n} / x_{n}\right), u^{2}\left(x_{n+1}^{*}\right)=1+O\left(\log x_{n} / x_{n}\right)$, we see from (2.6) that

$$
\begin{aligned}
v\left(x_{n}^{*}\right) & \sim \frac{1}{2}(1-\sqrt{1-4 L}), \\
v\left(x_{n+1}^{*}\right) & \sim \begin{cases}\frac{1}{2}(1+\sqrt{1-4 L}) & \text { for } L>0, \\
\frac{1}{2}(-1-\sqrt{1-4 L}) & \text { for } L<0 .\end{cases}
\end{aligned}
$$

Thus, integrating (2.7) over $\left(x_{n}^{*}, x_{n+1}^{*}\right)$, we have

$$
\begin{aligned}
& t_{n+1}^{*}-t_{n}^{*}=2 \log \frac{1+\sqrt{1-4 L}}{|1-\sqrt{1-4 L}|}+o(1), \\
& \frac{x_{n+1}^{*}}{x_{n}^{*}}\{1+o(1)\}=\frac{(1+\sqrt{1-4 L})^{4}}{16 L^{2}}=a_{L},
\end{aligned}
$$


say, so that

$$
\frac{x_{n+1}}{x_{n}}=a_{L}\{1+o(1)\} \quad \text { as } \quad n \rightarrow \infty .
$$

We now want to make the estimate (2.6) more precise. We note that for $x$ sufficiently large,

$$
y^{2}-x \leq-\log x+K
$$

for some constant $K$, from (1.13), so that

$$
y^{2}-x+1 \text { is ultimately negative. }
$$

We now show that

$$
\int_{x_{n}}^{x_{n+1}}\left|y^{2}-x+1\right| e^{y^{2}-x} d x=O\left(\frac{\log x_{n}}{x_{n}}\right) .
$$

For since, at $x_{n}$, from (2.1),

$$
y^{2} \sim x-\log x+\log \left(\frac{1}{4}-L\right)
$$

we can divide the interval $\left(x_{n}, x_{n+1}\right)$ into three subsets:

$$
\begin{array}{ll}
S_{1} \quad \text { where } y^{2}-x \geq-2 \log x, \quad y \geq 0 \\
S_{2} \text { where } y^{2}-x \geq-2 \log x, \quad y<0 \\
S_{3} \text { where } y^{2}-x \leq-2 \log x .
\end{array}
$$

From $S_{1}$, we have the contribution

$$
O\left\{\int_{S_{1}} \log x e^{y^{2}-x} d x\right\}=O\left\{\int_{S_{1}} \log x\left(\frac{y}{\sqrt{x}}\right) e^{y^{2}-x} d x\right\}=O\left\{\frac{\log x_{n}}{\sqrt{x_{n}}} \int_{S_{1}} y e^{y^{2}-x} d x\right\} .
$$

From (1.1) and the fact that $y^{\prime}=O(1 / \sqrt{x})$, we see that the contribution from $S_{1}$ is of the required form, and $S_{2}$ is similar. In $S_{3}$, we have

$$
\left|y^{2}-x+1\right| e^{y^{2}-x}=O\left(\log x / x^{2}\right),
$$

so that

$$
\int_{S_{3}}\left|y^{2}-x+1\right| e^{y^{2}-x} d x=O\left(\log x_{n} / x_{n}\right)
$$

This proves (2.9).

Now, integrating $(1.9)$ over $(x, \infty)$, we have

$$
\left|(u-v) v-x e^{y^{2}-x}-L\right|=O\left(\sum_{N}^{\infty} \frac{\log x_{r}}{x_{r}}\right)
$$

where $x_{N}$ is the maximum of $H$ next below $x$. Since (2.7) certainly implies that $x_{N+p} \geq A^{p} x_{N}$, for some $A>1$, we see that in (2.10), we have

$$
\begin{gathered}
\frac{\log x_{r}}{x_{r}} \leq \frac{(r-N) \log A+\log x_{N}}{A^{r-N} x_{N}}, \\
\sum_{N}^{\infty} \frac{\log x_{r}}{x_{r}}=O\left(\frac{\log x_{N}}{x_{N}}\right)=O\left(\frac{\log x}{x}\right) .
\end{gathered}
$$

Hence,

$$
\left|(u-v) v-x e^{y^{2}-x}-L\right|=O(\log x / x),
$$

and this is the more precise form of (2.6). From this, (1.14) is easily derived. 
If we now follow through the argument that succeeds (2.6), with the more precise form of (2.6), we improve (2.8) to

$$
\frac{x_{n+1}}{x_{n}}=a_{L}\left\{1+O\left(\frac{\log x_{n}}{x_{n}}\right)\right\} .
$$

Since

$$
\prod^{\infty}\left(1+\frac{\log x_{n}}{x_{n}}\right)<\infty
$$

we conclude that

$$
x_{n}=k a_{L}^{n}\left\{1+O\left(n a_{L}^{-n}\right)\right\} .
$$

The remaining statements in the theorem are now trivial, except for the existence of solutions corresponding to any $L$.

To prove the existence of such solutions, we observe first that the proof of (2.9) is independent of the value of $L$, and that, in particular, the constant implicit in the order symbol in (2.9) is independent of $L$. Indeed, by integration of (1.9), what the proof actually shows is that, if $X_{1}, X_{2}\left(X_{2}>X_{1}\right)$ are two (large) values of $x$ between which $H$ has only a bounded number of maxima, say 2 , and if

$$
f(x)=(u-v) v-x e^{y^{2}-x},
$$

then

$$
\left|f\left(X_{2}\right)-f\left(X_{1}\right)\right|=O\left(\frac{\log X_{1}}{X_{1}}\right)
$$

say $\left|f\left(X_{2}\right)-f\left(X_{1}\right)\right|<K_{0} \frac{\log X_{1}}{X_{1}}, K_{0}$ independent of $L$. (The modulus signs can in fact be dropped since $f$ is ultimately increasing.) Further, the number $a_{L}(>1)$ is bounded from 1 so long as $L\left(<\frac{1}{4}\right)$ is bounded from $\frac{1}{4}$, and by effectively repeating the argument that leads to (2.8), we can easily show that, if $f\left(X_{2}\right)$ is bounded from $\frac{1}{4}$, say $\frac{1}{4}-f\left(X_{2}\right) \geq \eta$, and if $X_{2}$ and $X_{1}$ are separated by at least two maxima of $H$, then

$$
X_{2} / X_{1} \geq M(\eta)
$$

where $M(\eta)$ is bounded from 1 so long as $\eta$ is bounded from 0 .

Now take any fixed value of $L$, say $L_{0}, L_{0}<\frac{1}{4}$. Take a fixed $\eta$, with $0<\eta \leq$ $\frac{1}{2}\left(\frac{1}{4}-L_{0}\right)$. Choose a sequence of points $X_{n}, X_{1}$ being large and to be specified more precisely later and each $X_{n}$ separated from the next by two maxima of $H$. Choose a solution such that

$$
L_{0}-\frac{1}{2} \eta \leq f\left(X_{1}\right) \leq L_{0}+\frac{1}{2} \eta
$$

Then we shall prove inductively that

$$
f\left(X_{n}\right) \leq \frac{1}{4}-\eta
$$

and so

$$
X_{n} / X_{n-1} \geq M(\eta) \quad, \quad X_{n} \geq\{M(\eta)\}^{n-1} X_{1}
$$


To prove (2.14) (which is certainly true for $n=1$ ), we suppose it true up to $n-1$ and note that, by (2.11), (2.12), and (2.13),

$$
\begin{aligned}
f\left(X_{n}\right) & \leq f\left(X_{1}\right)+K_{0} \sum_{r=1}^{n-1} \frac{\log X_{r}}{X_{r}} \\
& \leq f\left(X_{1}\right)+K\left(K_{0}, M\right) \frac{\log X_{1}}{X_{1}}
\end{aligned}
$$

by a calculation similar to that in (2.11), $K$ being some constant that depends on $K_{0}$ and $M$ (and thus $\eta$ ). Since $X_{1}$ is still at our choice, we can choose it so large that (2.15) implies (2.14), and this induction proof is complete.

By taking the limit in (2.15) as $n \rightarrow \infty$, we see that we can arrange that $f\left(X_{n}\right)$ for all $n$, and thus the $\operatorname{limit} L=\lim _{n \rightarrow \infty} f\left(X_{n}\right)$, is as close as we please to $L_{0}$. Since $L_{0}<\frac{1}{4}$ is arbitrary, we can obtain solutions with values of $L$ which are arbitrarily large and negative or as close as we please to $\frac{1}{4}$.

To show that we can obtain any $L$ with $-\infty<L<\frac{1}{4}$, we have only to show that the limit $L$ is continuous in the initial data. Give any solution with initial data $\left(u_{0}, v_{0}\right)$ and limit $L_{0}$, we can certainly find $X_{0}$ sufficiently large that, given any $\eta$ as above,

$$
L_{0}-\frac{1}{4} \eta \leq f_{0}\left(X_{0}\right) \leq L_{0}+\frac{1}{4} \eta
$$

where the subscript in $f_{0}$ indicates that we are dealing with the solution with initial data $\left(u_{0}, v_{0}\right)$. If $\left(u_{1}, v_{1}\right)$ is sufficiently close to $\left(u_{0}, v_{0}\right)$, then certainly

$$
L_{0}-\frac{1}{2} \eta \leq f_{1}\left(X_{0}\right) \leq L_{0}+\frac{1}{2} \eta
$$

and so, from the argument above, the limit $L_{1}$ is close to $L_{0}$, proving the required continuity.

\section{Proof of Theorem 2}

To show the existence of solutions converging to $(0,0)$, we solve the integral equation

$$
y=A-\int_{x}^{\infty}(t-x) y e^{y^{2}-t} d t
$$

by iteration in the usual manner. This solution clearly has the asymptotic form

$$
y=A+O\left(e^{-x}\right)
$$

and $(u, v) \rightarrow(0,0)$.

In fact, any solution such that $(u, v) \rightarrow(0,0)$ must have $y \rightarrow A$, for some $A$. For $u \rightarrow 0$ certainly implies that

$$
y^{\prime \prime}=O\left(e^{-x}\right),
$$

and since $v \rightarrow 0$ implies $y^{\prime} \rightarrow 0$, we have

$$
y^{\prime}=O\left(e^{-x}\right), \quad y=A+O\left(e^{-x}\right) .
$$

The fact that $d\left\{(u, v), C_{0}\right\} \rightarrow 0$ for any solution is proved as for the corresponding result in Theorem 1 , and we do not repeat it. We show now that, if a solution does not converge to $(0,0)$, then necessarily, for $x$ sufficiently large, when the solution is close to the $u$-axis at the right-hand half of $C_{0}, v$ must become negative, thus forcing the solution then to follow the left-hand half of $C_{0}$. (This shows that any solution converges either to $(0,0)$ or to the whole of $C_{0}$.) 
To do this, suppose for contradiction that $v>0$. Now

$$
u v-v^{2}-x e^{y^{2}-x}<0
$$

since the left-hand side is increasing to zero, and so, if $v$ is small and positive and $u$ lies in the range $(2 v, 1-\delta)$, for some fixed $\delta>0$, we must have

$$
u v \leq 2 x \exp \left[-x\left(2 \delta-\delta^{2}\right)\right]
$$

From (1.6), with $t=\log x$, we have

$$
\begin{gathered}
u \frac{d u}{d t}+\frac{1}{2} u^{2}=u v, \\
\frac{d}{d t}\left(e^{t} u^{2}\right)=2 e^{t} u v .
\end{gathered}
$$

Since, for large $x$,

$$
\int_{x}^{\infty} s^{2} \exp \left[-s\left(2 \delta-\delta^{2}\right)\right] d s=O\left(x^{2} \exp \left[-x\left(2 \delta-\delta^{2}\right)\right]\right),
$$

we see that $x u^{2}$ remains essentially constant, so that $u$ becomes small. However, since $u$ is of order $1 / \sqrt{x},(3.1)$ implies that $v<\frac{1}{2} u$, and so $u$ never reaches the lower end of the range $(2 v, 1-\delta)$. Thus $(3.1)$ continues to hold, and we are forced to the conclusion that $(u, v) \rightarrow(0,0)$, which we have excluded. This contradiction gives us what we require.

All that remains is to exhibit the existence of a solution with $L=0$ and limit set given by the whole of $C_{0}$. To do this, take any large value $x_{0}$ and consider the solution $y_{k}$ or $\left(u_{k}, v_{k}\right)$ determined by

$$
y_{k}\left(x_{0}\right)=0, \quad y_{k}^{\prime}\left(x_{0}\right)=k \text {. }
$$

If $k(>0)$ is sufficiently large, then $y_{k}$ oscillates infinitely often. This is because the quantity

$$
u v-v^{2}-x e^{y^{2}-x}
$$

is large and negative at $x_{0}$, and since we know from our considerations in Theorem 1 that its total increase is only $O\left(\log x_{0} / x_{0}\right)$, it remains negative as $x \rightarrow \infty$, so that the corresponding value of $L$ has $L<0$. This implies infinitely many oscillations.

On the other hand, if $k(>0)$ is sufficiently small, then $y_{k}$ has no subsequent zeros. It is easy to see that the quantity (3.2) becomes positive (before $u$ or $v$ vanishes), and once it is positive (and increasing), we can never have $u=0$.

Thus let $k_{0}$ be the least value of $k$ such that, for $k>k_{0}$, the solution $y_{k}$ has at least one zero for $x>x_{0}$. It is clear that $y_{k_{0}}$ has no zeros for $x>x_{0}$, since if it had, so also would $y_{k}$ for all $k$ sufficiently close to $k_{0}$, even $k<k_{0}$. Thus $y_{k_{0}}$ does not oscillate, and so the corresponding $L_{0}$ has $L_{0} \geq 0$.

For $k>k_{0}$, all solutions $y_{k}$ have at least one zero for $x>x_{0}$. As we have already seen, if $k$ is sufficiently large, $y_{k}$ has infinitely many zeros. Let $k_{1}$ be the least value of $k$ such that, for $k>k_{1}$, the solution $y_{k}$ has at least two zeros for $x>x_{0}$. Now $k_{1} \neq k_{0}$, for if that were so, then, for $k>k_{1}$ and close to $k_{1}$, we would have two zeros, say $x_{1}, x_{2}$, both going to infinity as $k \downarrow k_{1}$. But if $x_{1} \rightarrow \infty$, then necessarily $y\left(x_{1}\right)=0$ and $y^{\prime}\left(x_{1}\right)$ is small, and, as we have already seen, this would imply that there are no subsequent zeros and that $x_{2}$ does not exist.

So $k_{1} \neq k_{0}$. The solution $y_{k_{1}}$ has precisely one zero and $L_{1} \geq 0$. Inductively, let $k_{n}$ be the least value of $k$ such that, for $k>k_{n}$, the solution $y_{k}$ has at least $n$ zeros for $x>x_{0}$, and consider $k^{*}=\lim _{n \rightarrow \infty} k_{n}$. Certainly, $k^{*}$ is finite, and since $L_{n} \geq 0$ for all 
$n$, we must have that $L^{*}$ (the value of $L$ corresponding to $k^{*}$ ) satisfies $L^{*} \geq 0$. But for any $k>k^{*}$, the corresponding solution $y_{k}$ has infinitely many zeros, and so $L<0$, $L^{*} \leq 0$. The conclusion is that $L^{*}=0$, and we are done.

\section{Proof of Theorem 3}

That the limit set $C_{1 / 4}$ consists of just the two points $\left(1, \frac{1}{2}\right)$ and $\left(-1,-\frac{1}{2}\right)$ is obvious.

We next show that any solution of (1.1) in the case when $L=1 / 4$ has the property that

$$
x\left(u^{2}-1\right)=-2 \log x-2 \log 2+\phi(x)
$$

where $\phi(x) \rightarrow 0$ as $x \rightarrow \infty$. The first part of the proof consists in proving this and in improving it until we reach the estimate (4.16). We are then in a position to convert the equation into an integral equation and complete the proof of the theorem.

To prove $\phi \rightarrow 0$, recall that we have the two equations

$$
\begin{gathered}
y^{2}+e^{y^{2}-x}=\int_{x}^{\infty} e^{y^{2}-t} d t \\
\left(v-\frac{1}{2} u\right)^{2}+\frac{1}{4}\left(1-u^{2}\right)+x e^{y^{2}-x}=\int_{x}^{\infty}\left(1-u^{2}-\frac{1}{t}\right) t e^{y^{2}-t} d t .
\end{gathered}
$$

From (4.3), it follows (as we saw earlier) that $x e^{y^{2}-x}$ is bounded, so that, for some constant $K$,

$$
x\left(u^{2}-1\right) \leq-\log x+K
$$

Following the same argument as in $\S 2$, when we were dealing with (2.9), we can show that

$$
\int_{x}^{\infty}\left(1-u^{2}\right) t e^{y^{2}-t} d t=O(\log x / x)
$$

(Divide the interval of integration into regions where $t\left(1-u^{2}\right) \leq-2 \log t$ and $t\left(1-u^{2}\right)>$ $-2 \log t$.) Thus, from (4.3),

$$
e^{y^{2}-x}=O\left(\log x / x^{2}\right)
$$

and

$$
v-\frac{1}{2} u=O\left(\log ^{1 / 2} x / x^{1 / 2}\right)
$$

which implies that

$$
v-\frac{1}{2}=O\left(\log ^{1 / 2} x / x^{1 / 2}\right)
$$

and that

$$
y^{\prime}=\frac{1}{2 x^{1 / 2}}+O\left(\frac{\log ^{1 / 2} x}{x}\right)
$$

From (4.2), we then have

$$
\int_{x}^{\infty} e^{y^{2}-t} d t=\frac{1}{4 x}+O\left(\frac{\log ^{\frac{1}{2}} x}{x^{3 / 2}}\right)
$$

and by an integration by parts

$$
\int_{x}^{\infty} \log t e^{y^{2}-t} d t=\frac{\log x}{4 x}+\frac{1}{4 x}+O\left(\frac{\log ^{3 / 2} x}{x^{3 / 2}}\right) .
$$


Note also that (4.5) can be written in the form

$$
\int_{x}^{\infty} \frac{1}{4 t^{2}}\left(e^{\phi}-1\right) d t=O\left(\frac{\log ^{\frac{1}{2}} x}{x^{3 / 2}}\right)
$$

Now substitute (4.1) in (4.3) to obtain

$$
\begin{aligned}
\left(v-\frac{1}{2} u\right)^{2}+ & \frac{1}{4 x}(2 \log x+2 \log 2-\phi)+\frac{1}{4 x} e^{\phi} \\
& =\int_{x}^{\infty}(2 \log t+2 \log 2-\phi-1) e^{y^{2}-t} d t \\
& =\frac{\log x}{2 x}+\frac{1}{2 x}+\frac{2 \log 2-1}{4 x}-\int_{x}^{\infty} \frac{1}{4 t^{2}} \phi e^{\phi} d t+O\left(\frac{\log ^{3 / 2} x}{x^{3 / 2}}\right) .
\end{aligned}
$$

In view of (4.6), we can rewrite this as

$$
\left(v-\frac{1}{2} u\right)^{2}+\frac{1}{4 x}\left(e^{\phi}-\phi-1\right)=\int_{x}^{\infty} \frac{1}{4 t^{2}}\left(e^{\phi}-1-\phi e^{\phi}\right)+O\left(\frac{\log ^{\frac{3}{2}} x}{x^{\frac{3}{2}}}\right) .
$$

But

$$
e^{\phi}-\phi-1 \geq 0 \quad \text { for all } \quad \phi
$$

and

$$
e^{\phi}-1-\phi e^{\phi} \leq 0 \quad \text { for all } \quad \phi,
$$

with equality in both cases only when $\phi=0$. So we conclude from (4.7) that

$$
v-\frac{1}{2} u=O\left(\frac{\log ^{3 / 4} x}{x^{3 / 4}}\right)
$$

and that (since $e^{\phi}-\phi-1$ is precisely of order $\phi^{2}$ for small $\phi$ )

$$
\phi=O\left(\frac{\log ^{3 / 4} x}{x^{1 / 4}}\right)
$$

Thus certainly $\phi \rightarrow 0$.

It is now easy to improve this. If we go back to (4.5) and use (4.8) in place of (4.4), we can repeat the argument and obtain

$$
\begin{gathered}
v-\frac{1}{2} u=O\left(\frac{\log ^{7 / 8} x}{x^{7 / 8}}\right), \\
\phi=O\left(\frac{\log ^{7 / 8} x}{x^{3 / 8}}\right) .
\end{gathered}
$$

Indeed, by successive repetition, we could reach

$$
v-\frac{1}{2} u=O\left(\frac{\log ^{1-\eta} x}{x^{1-\eta}}\right), \phi=O\left(\frac{\log ^{1-\eta} x}{x^{\frac{1}{2}-\eta}}\right),
$$

for any $\eta>0$, but we have to do better still, which requires looking at the error terms more closely.

Since we now have (4.10), we can write

$$
\begin{aligned}
y^{\prime 2} & =\frac{1}{x}\left(\frac{1}{2}+\left(v-\frac{1}{2}\right)\right)^{2} \\
& =\frac{1}{4 x}+\frac{v-\frac{1}{2}}{x}+O\left(x^{-2-\delta}\right),
\end{aligned}
$$


for some $\delta>0$, not necessarily the same at each appearance. From (4.1) and (4.11),

$$
e^{y^{2}-x}=\frac{1}{4 x^{2}}+O\left(\frac{\log ^{7 / 8} x}{x^{19 / 8}}\right) .
$$

Using (4.2), (4.12), and the last formula, we see that (4.5) becomes

$$
\int_{x}^{\infty} e^{y^{2}-t} d t=\frac{1}{4 x}+\frac{v-\frac{1}{2}}{x}+\frac{1}{4 x^{2}}+O\left(x^{-2-\delta}\right)
$$

and

$$
\begin{aligned}
\int_{x}^{\infty} \log t e^{y^{2}-t} d t= & \frac{\log x}{4 x}+\frac{\left(v-\frac{1}{2}\right) \log x}{x}+\frac{\log x}{4 x^{2}}+O\left(x^{-2-\delta}\right) \\
& +\int_{x}^{\infty} \frac{1}{t}\left\{\frac{1}{4 t}+\frac{v-\frac{1}{2}}{t}+\frac{1}{4 t^{2}}\right\} d t \\
= & \frac{\log x}{4 x}+\frac{1}{4 x}+\frac{\left(v-\frac{1}{2}\right) \log x}{x}+\frac{\log x}{4 x^{2}}+\frac{1}{8 x^{2}}+O\left(x^{-2-\delta}\right) \\
& +\int_{x}^{\infty} \frac{v-\frac{1}{2}}{t^{2}} d t
\end{aligned}
$$

But

$$
x(u-1)^{\prime}=v-\frac{1}{2}-\frac{1}{2}(u-1)
$$

so that

$$
\begin{aligned}
\int_{x}^{\infty} \frac{v-\frac{1}{2}}{t^{2}} d t & =\int_{x}^{\infty} \frac{(u-1)^{\prime}}{t} d t+\frac{1}{2} \int_{x}^{\infty} \frac{u-1}{t^{2}} d t \\
& =-\frac{u-1}{x}+\frac{3}{2} \int_{x}^{\infty} \frac{u-1}{t^{2}} d t \\
& =\frac{\log x}{4 x^{2}}+\frac{\log 2}{4 x^{2}}-\frac{3}{8 x^{2}}+O\left(x^{-2-\delta}\right),
\end{aligned}
$$

by using (4.1) and (4.11). If we substitute (4.13), (4.14), (4.15) in (4.3), and write

$$
\begin{aligned}
\left(v-\frac{1}{2} u\right)^{2} & =\left(v-\frac{1}{2}\right)^{2}-\left(v-\frac{1}{2}\right)(u-1)+\frac{1}{4}(u-1)^{2} \\
& =\left(v-\frac{1}{2}\right)^{2}+\left(v-\frac{1}{2}\right) \frac{\log (2 x)}{x}+\frac{1}{4}\left(\frac{\log (2 x)}{x}\right)^{2}+O\left(x^{-2-\delta}\right),
\end{aligned}
$$

we obtain, after some algebra,

$$
\begin{aligned}
& \left\{v-\frac{1}{2}-\frac{1}{2 x}(\log (2 x)-2)\right\}^{2}+\frac{1}{4 x}\left(e^{\phi}-\phi-1\right)-\int_{x}^{\infty} \frac{1}{4 t^{2}}\left(e^{\phi}-1-\phi e^{\phi}\right) d t \\
& =O\left(x^{-2-\delta}\right),
\end{aligned}
$$

whence

$$
\begin{gathered}
v-\frac{1}{2}=\frac{\log (2 x)}{2 x}-\frac{1}{x}+O\left(x^{-1-\delta}\right), \\
\phi=O\left(x^{-\frac{1}{2}-\delta}\right) .
\end{gathered}
$$

We now write the equation (1.1) in terms of the function $g$, defined by

$$
g=u-1+\frac{\log x}{x}+\frac{\log 2}{x} \text {. }
$$


From the estimates (4.16) on $\phi$, we have the estimate

$$
g=O\left(x^{-\frac{3}{2}-\delta}\right)
$$

It is easy to verify that $g$ satisfies

$$
x^{2} g^{\prime \prime}+x g^{\prime}=x^{2} G^{\prime \prime}+x G^{\prime}+\left\{\frac{1}{4}-\frac{1}{4} e^{2 x g+x(u-1)^{2}}\right\} u
$$

where

$$
G(x)=\frac{\log (2 x)}{x}
$$

and we can rewrite (4.18) as

$$
\begin{aligned}
& x^{2} g^{\prime \prime}+x g^{\prime}+\frac{1}{2} x g=x^{2} G^{\prime \prime}+x G^{\prime} \\
& \quad+\frac{1}{4}\left\{-(1+2 x g)\left(e^{x(u-1)^{2}}-1\right)-e^{x(u-1)^{2}}\left(e^{2 x g}-1-2 x g\right)\right\} u \\
& \quad+\frac{1}{2} x g(1-u)=F
\end{aligned}
$$

say. Since

$$
x^{2} g^{\prime \prime}+x g^{\prime}+\frac{1}{2} x g=0
$$

has solutions

$$
g=J_{0}(\sqrt{2 x}), Y(\sqrt{2 x}),
$$

where $Y$ is a second solution of the equation chosen so that

$$
W\left(J_{0}, Y\right)=\frac{1}{x}
$$

and since $J_{0}$ has the asymptotic expression

$$
J_{0}(z)=\sqrt{\frac{2}{\pi z}} \cos \left(z-\frac{1}{4} \pi\right)+O\left(z^{-3 / 2}\right),
$$

with a similar expression for $Y$, we can write (4.19) in the integral form

$$
g=\int_{x}^{\infty}\left\{J_{0}(\sqrt{2 x}) Y(\sqrt{2 t})-J_{0}(\sqrt{2 t}) Y(\sqrt{2 x})\right\} \frac{F}{t} d t
$$

Since, for $t \geq x$, we have

$$
J_{0}(\sqrt{2 x}) Y(\sqrt{2 t})-J_{0}(\sqrt{2 t}) Y(\sqrt{2 x})=-\frac{2}{x^{1 / 4} t^{1 / 4}} \sin (\sqrt{x}-\sqrt{t})+O\left(x^{-3 / 4} t^{-1 / 4}\right),
$$

there is no difficulty from the form of $F$ and the estimate (4.17) on $g$ in asserting that the infinite integral converges. Also, we do not need to include in (4.20) arbitrary multiples of $J_{0}(\sqrt{2 x}), Y(\sqrt{2 x})$ since the estimate on $g$ necessarily excludes them.

We are going to look for solutions of (4.20) in the space $B$ of continuous functions $g$ such that, for some fixed $\delta>0$,

$$
g=O\left(x^{-3 / 2-\delta}\right)
$$

(The earlier estimates would have given us a specific choice of $\delta$, but this is unimportant.) We want to show first that the integral operator on the right side of (4.20) 
carries $B$ into itself, and so we write

$$
\begin{gathered}
u-1=g-\frac{\log (2 x)}{x}, \\
x(u-1)^{2}=\frac{\log ^{2}(2 x)}{x}+O(g \log x)+O\left(x g^{2}\right), \\
F=x^{2} G^{\prime \prime}+x G^{\prime}-\frac{1}{4} \frac{\log ^{2}(2 x)}{x}+O\left(x^{2}(u-1)^{4}\right)+O\left(x^{2} g(u-1)^{2}\right) \\
+O\left(x^{2} g^{2}\right)+O(x g(1-u)) .
\end{gathered}
$$

Of the first three terms in (4.22), the major one is the third, and if we substitute this on the right side of (4.20), and use (4.21) and integrate by parts, we obtain a contribution to the right side of $(4.20)$ which is certainly of order $O\left(x^{-3 / 2-\delta}\right)$, as required. (The integration by parts is required only on the trigonometric term in (4.21); the O-term gives no problems.) For the O-terms in (4.22), if $g \in B$, we also see easily that the result of the operation in (4.20) is again in $B$. (Here the integration by parts is not required.)

It is routine to show that the mapping is in fact a contraction mapping, the details being left to the reader. Thus there exists a unique solution of (4.20) (and so of (4.18)) in $B$, and the proof is complete.

We now can use (4.20) to develop a complete asymptotic expansion of the solution, but we do not pursue this.

Acknowledgements. The work of the second author was supported by NSF Grant DMS95-01395.

\section{References}

1. F. V. Atkinson and S. Chen, On the nonoscillation of an Emden-Fowler equation, Canad. Math. Soc. Conf. Proc. 8 (1987), 43-55.

University of Pittsburgh, Department of Mathematics, Pittsburgh, PA 15260 